\title{
Acute neuropsychological effects of MDMA and ethanol (co-) administration in healthy volunteers
}

\author{
G. J. H. Dumont • E. Wezenberg • \\ M. M. G. J. Valkenberg • C. A. J. de Jong • \\ J. K. Buitelaar • J. M. A. van Gerven • R. J. Verkes
}

Received: 13 June 2007 / Accepted: 19 December 2007 /Published online: 28 February 2008

(C) The Author(s) 2008

\begin{abstract}
Rationale In Western societies, a considerable percentage of young people expose themselves to 3,4-methylenedioxymethamphetamine (MDMA or "ecstasy"). Commonly, ecstasy is used in combination with other substances, in particular alcohol (ethanol). MDMA induces both arousing as well as hallucinogenic effects, whereas ethanol is a general central nervous system depressant.

Objective The aim of the present study is to assess the acute effects of single and co-administration of MDMA and ethanol on executive, memory, psychomotor, visuomotor, visuospatial and attention function, as well as on subjective experience. Materials and methods We performed a four-way, doubleblind, randomised, crossover, placebo-controlled study in 16 healthy volunteers (nine male, seven female) between the ages of 18-29. MDMA was given orally $(100 \mathrm{mg})$ and blood alcohol concentration was maintained at $0.6 \%$ by an ethanol infusion regime.
\end{abstract}

G. J. H. Dumont • E. Wezenberg • M. M. G. J. Valkenberg •

J. K. Buitelaar $\cdot$ R. J. Verkes

Unit for Clinical Psychopharmacology and Neuropsychiatry (UCPN),

Department of Psychiatry,

University Medical Centre St Radboud,

Nijmegen, The Netherlands

J. M. A. van Gerven

Centre for Human Drug Research (CHDR),

Leiden, The Netherlands

C. A. J. de Jong

Nijmegen Institute for Science Practitioners in Addiction, Radboud University Nijmegen,

Nijmegen, The Netherlands

G. J. H. Dumont $(\bowtie)$

University Medical Centre Nijmegen,

P.O. Box 9101, 6500 HB Nijmegen, The Netherlands

e-mail: G.J.H.Dumont@psy.umcn.nl
Results Co-administration of MDMA and ethanol was well tolerated and did not show greater impairment of performance compared to the single-drug conditions. Impaired memory function was consistently observed after all drug conditions, whereas impairment of psychomotor function and attention was less consistent across drug conditions.

Conclusions Co-administration of MDMA and ethanol did not exacerbate the effects of either drug alone. Although the impairment of performance by all drug conditions was relatively moderate, all induced significant impairment of cognitive function.

Keywords MDMA · Ecstasy - Alcohol E Ethanol · Interaction $\cdot$ Acute $\cdot$ Effects $\cdot$ Healthy volunteers . Neuropsychologic

\section{Introduction}

In Western societies, a considerable proportion of young people expose themselves to 3,4-methylenedioxymethamphetamine (MDMA or 'ecstasy'; Gross 2002; Parrott 2001; Tancer and Johanson 2007). Ecstasy has gained widespread use in the 'club' scene, typically all-night parties with loud music and intense lights (Winstock et al. 2001). The average dose of ecstasy used recreationally is reported to be around 80-90 mg of MDMA with considerable individual variation (Tanner-Smith 2006). Ecstasy users are generally multidrug users who have experience with various recreational drugs and use these in combination with ecstasy (Gouzoulis-Mayfrank and Daumann 2006b). Probably due to its availability, alcohol remains one of the most co-used substances (Barrett et al. 2005). As the use of alcohol is known to induce impairment of cognitive function and decrease the awareness of this impairment, 
this can lead to dangerous behaviour like driving under influence (Lamers and Ramaekers 2001; Riley et al. 2001).

MDMA acts primarily by releasing serotonin (5hydroxytryptamine (HT)) from pre-synaptic 5-HT terminals. It reverses the direction of the reuptake transporter and increases 5-HT levels at the post-synaptic receptors (Liechti and Vollenweider 2000; Mlinar and Corradetti 2003; Pifl et al. 1995). MDMA is also a potent releaser of dopamine and (nor)adrenaline (Colado et al. 2004; Liechti and Vollenweider 2001).

MDMA is rapidly absorbed following oral administration. Within $30 \mathrm{~min}$, MDMA is detectable in the blood. Plasma levels peak at $1-2 \mathrm{~h}$ after drug administration, and maximum behavioural and subjective effects occur around $1-2 \mathrm{~h}$ and have declined by $4 \mathrm{~h}$ in spite of persisting plasma levels (de la Torre et al. 2004; Green et al. 2003). Increasing the dose does not result in a proportional rise in plasma concentrations, which is indicative of non-linear pharmacokinetics (de la Torre et al. 2000).

The behavioural effects of MDMA resemble but are not restricted to effects of psychostimulants (e.g. amphetamines or 'speed') as well as hallucinogenics (e.g. lysergic acid or 'lysergic acid diethylamide'), although MDMA's most characteristic effects are described as an increase in empathy and friendliness (Vollenweider et al. 2002). This led to MDMA being categorized as an 'entactogen,' as coined by Nichols and Oberlender (1990).

Most research into the cognitive effects of MDMA in humans has focused on the long-term effects, where only memory was consistently found to be impaired (Verbaten 2003; Verkes et al. 2001). Our review of the acute effects of MDMA in humans showed that cognitive effects were assessed only in a limited number of studies, using diverse tests and generally addressing only certain aspects of neuropsychological function. As such, no consensus on MDMA's cognitive effects could be reached (Dumont and Verkes 2006). Since then, reports on the effects of MDMA generally confirmed previous findings (Kuypers et al. 2006; Kuypers et al. 2007; Ramaekers et al. 2006; Tancer and Johanson 2007). Interestingly, two studies reported effects of MDMA on memory, which had not been assessed previously. These reports showed acute impairment of immediate and delayed recall of words as well as spatial memory by MDMA (Kuypers and Ramaekers 2005, 2007).

Drinks containing ethanol, commonly referred to as alcohol, are widely available and regularly used in Western society. Ethanol is chiefly a central nervous system (CNS) depressant. It inhibits both excitatory and inhibitory postsynaptic potentials by potentiating the action of gammaaminobutyric acid at its receptor (Suzdak et al. 1988). Reports of the cognitive effects of combined use of MDMA and ethanol in humans have been sparse in the literature.
Studies that were performed assessed psychomotor function, attentional performance and subjective effects (HernandezLopez et al. 2002; Kuypers et al. 2006; Ramaekers et al. 2006). In general, MDMA and ethanol had no or opposite effects on effect measures, and as such co-administration did not exacerbate single-drug effects.

In the current study, we employed a series of tests sensitive to changes in all common neuropsychological domains induced by several pharmacological compounds, including amphetamines (Wezenberg et al. 2004).

It is generally acknowledged that the combined use of alcohol with other CNS-depressant drugs may enhance the effects of ethanol or of the other drugs. MDMA, however, has stimulant effects while ethanol is a sedative agent, suggesting that the effects of co-administration are diminished rather than augmented compared to the effects following single administration. This hypothesis was investigated during acute co-administration of MDMA and ethanol in healthy volunteers.

\section{Materials and methods}

Study design

This study utilised a four-way, double-blind, randomised, crossover, placebo-controlled design. Sixteen volunteers were randomly assigned to one of four treatment sequences. Each volunteer received a capsule containing either 100-mg MDMA or placebo and an ethanol-placebo infusion (target blood alcohol concentration (BAC) of $0.6 \%$ ) with a washout of 7 days between each treatment.

\section{Study outline}

Subjects arrived in the morning and were admitted to the study after a negative urine drug screen (opiates, cocaine, benzodiazepines, amphetamines, methamphetamines and delta-9-tetrahydrocannabinol), as well as a negative alcohol breath test and recording of signs and symptoms of possible health problems. A light breakfast was offered. Drug administration was scheduled at 1030 hours and the alcohol infusion was started at 1100 hours for a duration of $3 \mathrm{~h}$. At 1130 hours, subjects performed the psychological test battery as described below. Specific test times are reported in Table 1. Subjects received lunch at 1400 hours and were sent home at 1700 hours after a medical check. Adverse events where recorded throughout the study day. Vital signs were monitored using a Datascope ${ }^{\circledR}$ Accutorr Plus ${ }^{\mathrm{TM}}$ cardiovascular monitor and Braun ${ }^{\circledR}$ type 6021 ThermoScan during the study day. The data presented in this report are a subset of a larger data set, which will be reported elsewhere. 
Table 1 Timeline

\begin{tabular}{lll}
\hline Neuropsychological tests & Description & Time (h:m) \\
\hline Drug administration & & $0: 00$ \\
18-word list immediate recall & Immediate recall of 18-word list & $1: 00$ \\
SDST & Translate symbols to digits with key present in $90 \mathrm{~s}$ & $1: 05$ \\
SDRT & Translate symbols to digits from memory & $1: 08$ \\
Pursuit task & Keep dot within moving circle & $1: 10$ \\
Tangles task & Tangled line leads to which target? & $1: 13$ \\
Switch task & Follow, possibly conflicting, instructions (choice between left or right) \\
18 -word list delayed recall & Delayed recall of 18-word list & $1: 17$ \\
18 -word list delayed recognition & Recognise words of 18-word list memorised earlier among 18 distracters & $1: 22$ \\
Point task & Keep pen steady in air, measures tremor & $1: 23$ \\
Visual analogue scales & 16 100-mm scales for subjective experiences & $1: 25$ \\
\hline
\end{tabular}

Times are relative to drug administration.

\section{Subjects}

Sixteen healthy volunteers (nine male, seven female), regular users of ecstasy and alcohol, aged 18-29 years and within $80-130 \%$ of their ideal bodyweight, were recruited through advertisement on the internet and at local drug testing services. They were all in good physical and mental health as determined by assessment of medical history, a medical, electrocardiogramme and clinical, haematological and chemical blood examination. Previous drug use was assessed using a structured interview. Fifteen volunteers were right handed and one was left handed. The study was approved by the local Medical Ethics Committee. All subjects gave their written informed consent before participating in the study and were compensated for their participation. Subject demographics and drug history are reported in Table 2.

Table 2 Volunteer demographics-drug history

\begin{tabular}{lllll}
\hline & Mean & SD & Min & Max \\
\hline Age (years) & 22.1 & 2.9 & 18.0 & 29.0 \\
Education (years) & 16.5 & 1.6 & 12 & 18 \\
Height (cm) & 174.7 & 12.3 & 147.0 & 189.1 \\
Weight (kg) & 67.5 & 12.4 & 45.7 & 88.4 \\
Opiates & 0.1 & 0.3 & 0 & 1 \\
LSD & 2.5 & 6.6 & 0 & 25 \\
Amphetamines & 37.3 & 81.1 & 0 & 250 \\
Ecstasy & 94.6 & 138.4 & 14 & 431 \\
Cannabis & $1,174.3$ & $1,665.5$ & 20 & 5,840 \\
Cocaine & 33.7 & 105.7 & 0 & 400 \\
Alcohol & $2,367.9$ & $1,981.6$ & 50 & 5,200 \\
Solvents & 3.6 & 13.3 & 0 & 50 \\
Barbiturates & 0 & 0 & 0 & 0 \\
Benzodiazepines & 18.6 & 57.3 & 0 & 216 \\
Psilocybin & 6.9 & 10.4 & 0 & 30 \\
\hline
\end{tabular}

Drug quantities mentioned are lifetime drug exposures, not further specified.
One subject had a mild adverse reaction (local vascular reaction) to the alcohol infusion and one subject did not refrain from drug use; both (one male, one female) were excluded from further participation and results obtained were not included in the data analysis.

\section{Drugs and dosages}

MDMA (or matched placebo) was given as a capsule in a single dose of $100 \mathrm{mg}$ via oral administration (dose range; $1.1-2.2 \mathrm{mg} / \mathrm{kg}$ ). MDMA was obtained from Lipomed AG, Arlesheim, Switzerland and encapsuled according to Good Manufacturing Practise by the Department of Clinical Pharmacy, UMC St Radboud, Nijmegen, the Netherlands. MDMA $100 \mathrm{mg}$ orally is a relevant dose in the range of normal single recreational dosages. Previous experiments in humans used doses up to $150 \mathrm{mg}$ without serious adverse events. Ethanol (or matched placebo) was administered continuously by intravenous infusion of $10 \%$ ethanol in glucose solution resulting in an ethanol blood concentration of $0.6 \%$ with a duration of $3 \mathrm{~h}$ as described below.

\section{Alcohol clamping}

To standardise alcohol delivery and maintain a constant alcohol blood concentration over time, an intravenous ethanol clamp was used. Ethanol was administered by infusion of a $10 \%$ ethanol in glucose solution for a duration of $3 \mathrm{~h}$. The infusion rate was calculated using frequent breath alcohol concentrations measurements, according to a previously designed algorithm (Amatsaleh et al. 2006a). Breath alcohol concentration was assessed using a HONAC AlcoSensor IV ${ }^{\circledR}$ Intoximetre.

An intravenous administration route was chosen, ensuring standardisation of the rate and bioequivalence of ethanol administration. This is an important prerequisite for predictable pharmacokinetics of ethanol. The process was semi-automated using a computer 
spreadsheet programme, which uses measured breath alcohol concentrations to calculate the infusion rate needed to maintain the ethanol level at $0.6 \mathrm{mg} / \mathrm{mL}$. This is a relevant dose equivalent to peak levels of approximately two to three units of alcoholic beverages. In many European countries driving is prohibited at BAC above $0.5 \%$. This limit has been confirmed by a report that shows that at an average $\mathrm{BAC}$ of $0.6 \%$ psychomotor performance is significantly impaired (Amatsaleh et al. 2006b). A BAC of $0.6 \%$ is equivalent to approximately two to three alcoholic beverages commonly used in social settings in Western society, which is considered to be a safe and relatively moderate dose, despite its significant CNS effects.

\section{MDMA blood analysis}

For the assessment of serum levels of MDMA, blood samples were collected 90 min after drug administration from each subject on each study day. Venous blood samples $(10 \mathrm{ml})$ were collected into heparinised tubes, centrifuged immediately at $4^{\circ} \mathrm{C}$ for $15 \mathrm{~min}$. Plasma was split into aliquots of $2 \mathrm{~mL}$ (to prevent over-freezing-thawing), frozen rapidly using liquid nitrogen and stored at $-80^{\circ} \mathrm{C}$. Samples were analysed for MDMA and MDA concentration by the Toxicology unit of the Leyenburg hospital, The Hague, the Netherlands.

Neuropsychological tests, apparatus and procedure

The performance on all neuropsychological tests was recorded by means of a digitising tablet (WACOM UD1218-RE), a laptop computer, a pressure-sensitive pen (which could also be used as a cursor) and test forms. The $x$ and $y$ coordinates of the pen tip on and up to $5 \mathrm{~mm}$ above the digitiser were sampled with a frequency of $200 \mathrm{~Hz}$ and a spatial accuracy of $0.2 \mathrm{~mm}$. The time schedule of the tests is summarised in Table 1.

To familiarise the subjects with the tests and procedures, they were invited to the hospital to perform a practise session within 1 week before the actual study days. All tests had five equivalent versions for four test days and one practise day, test versions were counterbalanced over test days.

Executive function

Switch task This test is a reaction time task measuring simple as well as complex reaction time, assessing executive performance (Baker and Letz 1986). After a random period of 0.75 to $1.75 \mathrm{~s}$, two rectangular fields appeared on both sides of a circle in the centre of the screen. Only one of the two fields provided the subjects with information, either a colour, an arrow or both. The other non-informative field always had a neutral grey colour. Five conditions were subsequently presented to subjects. If only green fields appeared, subjects had to move as fast as possible into the green field. If green and red fields appeared, subjects had to move into the green field and away from the red field as soon as they appeared.

If green fields with a left or right arrow were presented, subjects were to move into the direction of the arrow. Green and red fields with a left or right arrow indicated that subjects were to follow the direction of the arrows in the green field, but the opposite direction of the arrows in the red field. Finally, the first condition was repeated. All conditions contained 20 trials except condition four in which there were 40 trials (total $=120$ trials). The outcome measures were the mean reaction times per condition. The last condition is a repetition of the first to check for possible changes in attention.

\section{Memory}

Eighteen-word list A verbal memory test based on the classic Auditory Verbal Learning Test (Vakil and Blachstein 1993) was used. A variant was made consisting of a list of 18 words. The classic test uses 15 words. A longer wordlist was chosen, however, to prevent ceiling effects. The list was presented verbally three times. Under normal circumstances, subjects are supposed to remember an increasing number of words after each trial. Directly after each presentation, and after an interval of $20 \mathrm{~min}$, subjects were asked to recall as many words as possible. After the delayed recall trial, a list of 36 words was presented from which they were asked to recognise the 18 words previously presented. The incorrect words were distracters and resembled the correct words in a semantic or phonologic manner. Responses were either correct positive (when a word that was recognised was indeed part of the list presented during immediate recall) or false positive (when a word was recognised but was not part of the list presented during immediate recall, e.g. the word was a distracter). The outcome measure was the number of correctly recalled or recognised words for the average of the three immediate recall trials, the delayed recall trial and the delayed recognition trial.

Symbol digit recall test The symbol digit recall test (SDRT) followed directly after the Symbol Digit Substitution test (SDST), which is discussed in the last paragraph of this section. After subjects had finished the SDST, they were shown the symbols of the SDST 
without the translation key, one at a time, and asked to produce the corresponding numbers. This test is based on an extended procedure of the SDST to measure incidental learning (Kaplan et al. 1991). The outcome measure was the number of correctly translated symbols.

\section{Psychomotor function}

Pursuit task To measure implicit procedural learning, a computerised version of the rotor pursuit task was used. This test is based on the classic rotary pursuit task (Ammons 1951). It is a continuous motor task. Subjects had to follow the movement of a large target stimulus on the computer screen with a cursor by moving the pen over the XY tablet. The speed of the target gradually increased when the cursor was contained within the target but decreased considerably when it was not. The target followed a spatially predictable circular path over the screen. The outcome measure for this test was the total number of rotations within $2 \mathrm{~min}$.

Point task The point task, a measure for tremor, required subjects to try to keep the cursor inside a very small circle for $1 \mathrm{~min}$, while avoiding contact between the pen and the test form. The outcome measure for this test was the deviation from the target.

\section{Visuospatial and visuomotor function}

Tangle task The tangle task required the subject to visually track a particular line winding through two to four other lines. On subsequent trials, the tangles increased in complexity; they got longer and made more $90^{\circ}$ turns. The paper form had a start area and five target areas, numbered 1 to 5 , which reflect the maximum target areas on the screen, starting with only three target areas.

This test is modelled after the visualisation test from the 'kit for factor referenced cognitive tests.' It was selected by the US NAVY to study environmental and other time-course effects and has good task stability and reliability (Bittner et al. 1986). The outcome measures are the reaction time per trial and the number of correct trials in $2 \mathrm{~min}$.

\section{Attention}

Symbol Digit Substitution test This test is a version of the subtest from the Wechsler Adult Intelligence Scale (Wechsler 1981). Subjects had to substitute the nine symbols for the digits 1-9 on the basis of a given translation key. The outcome measure was the total number of digits completed in $90 \mathrm{~s}$.

According to Hege et al. (1997) this task measures many cognitive components, e.g. visuospatial scanning, intermediate memory, perceptual motor speed and speed of cognitive processing. Therefore, subsequent analyses were performed in order to attempt and disentangle these cognitive processes. Based on pen pressure, movement trajectories were defined as either pen-up periods or pendown periods. This allowed for subsequent analysis of matching times and movement (writing) times in the Symbol Digit Substitution test. For the motor component, the mean writing times were computed. For the more cognitive component, the mean matching times were computed. These analyses have been previously performed (Sabbe et al. 1999; Wezenberg et al. 2005).

\section{Subjective}

Subjective effects were recorded using the Bond and Lader (Visual Analogue) Mood Rating Scale (BLMRS). This inventory was completed at the end of each neuropsychological test battery on each study day.

The BLMRS scale consisted of 16 lines, each $10 \mathrm{~cm}$ in length, with opposite terms at each end of the line (alert-drowsy, calm-excited, strong-feeble, muzzy-clearheaded, well coordinated-clumsy, lethargic-energetic, contented-discontented, troubled-tranquil, mentally slow-quick witted, tense-relaxed, attentive-dreamy, incompetent-proficient, happy-sad, antagonistic-amicable, interested-bored, withdrawn-gregarious). Subjects were asked to indicate which item was more appropriate by marking the line. The outcome measure was the distance to the marker on each scale. These scale scores were then aggregated to scores for 'calmness,' 'alertness' and 'contentedness' as described by Bond et al. (1974).

Statistical analyses

Statistical evaluation (using SPSS 11.5 for Windows) was performed with general linear model repeatedmeasures analysis of variance. Main and interaction effects were tested using a two-factor ('ethanol' and 'MDMA'), two-level (absent versus present) multivariate model.

The analysis of the data was based on Maxwell and Delaney (2004) and Kirk (1995). First the presence of interaction (non-additivity) was tested with alfa $=0.05$. When the interaction was not statistically significant we proceeded by testing the main effects, each at alfa $=0.05$. In the case of a significant interaction, we proceeded by 
testing simple main effects of each drug, i.e. MDMA vs. placebo and ethanol vs. placebo.

\section{Results}

Subject demographics are summarised in Table 2. Out of 16 subjects, 14 completed the study procedure. One subject had a mild adverse reaction (local vascular reaction which subsided with infusion stop) to the alcohol infusion and one subject did not refrain from drug use; both were discontinued from study participance and data already obtained were not included in statistical analysis. Only significant results are mentioned in this section, unless stated otherwise.

MDMA blood concentration 90 min after administration did not differ for MDMA single vs. MDMA and ethanol co-administration and was on average $196 \mu \mathrm{g} / \mathrm{L}(\mathrm{SD}=$ $83 \mu \mathrm{g} / \mathrm{L}$ ). Blood alcohol concentration was maintained at an average of $0.54 \%$ o $(\mathrm{SD}=0.07 \%)$.

\section{Executive function}

Executive function (switch task) did not show any significant main or interaction effects.

\section{Memory function}

Memory function was assessed by the 18-word list (outcome measures were 'immediate recall,' 'delayed recall' and 'recognition,' see Fig. 1) as well as the SDRT. Immediate recall was impaired only by ethanol $(F(1,12)=$ $8.71, p=0.011)$.

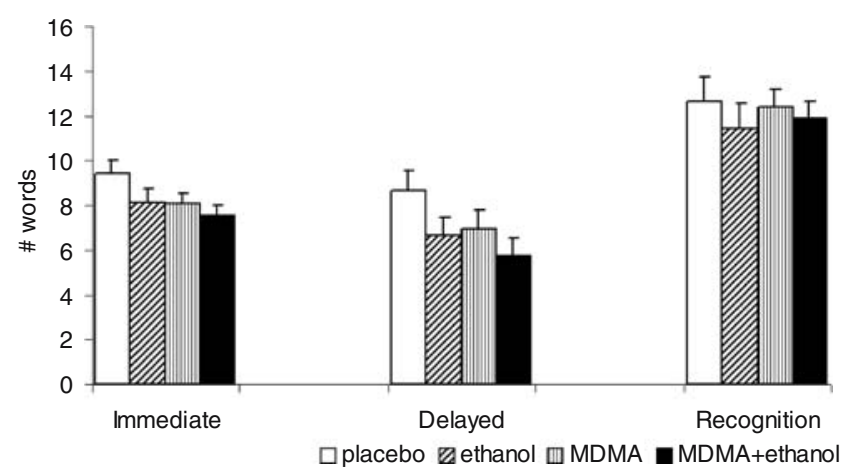

Fig. 1 Memory effects (18-word list), Immediate: immediate recall, average score of three trials of correctly recalled verbally presented words, Delayed: correctly recalled verbally presented words $20 \mathrm{~min}$ after presentation, Recognition: correctly recognised verbally presented words containing 18 distracters, $20 \mathrm{~min}$ after presentation (mean and SEM). Immediate recall was impaired only by ethanol $(F(1,12)=8.71, p=0.011)$. Delayed recall was impaired by MDMA $(F(1,12)=10.447, p=0.007)$ as well as by ethanol $(F(1,12)=16.031$, $p=0.002)$; recognition was not affected by any drug condition
Delayed recall as assessed by the 18-word list was impaired by MDMA $(F(1,12)=10.447, p=0.007)$ as well as by ethanol $(F(1,12)=16.031, p=0.002)$. The SDRT, also a test for delayed recall, showed a similar pattern of impairment by MDMA $(F(1,12)=5.300, p=0.038)$ as well as by ethanol $(F(1,12)=7.654, p=0.016)$.

\section{Psychomotor function}

Psychomotor function was assessed with tests for tremor (point task), accuracy (pursuit task) and speed (SDST motor time, see Fig. 2); other SDST results are reported in the section "Attention." Ethanol impaired psychomotor speed as reflected in the increase in SDST motor time $(F(1,12)=$ 9.295, $p=0.009$ ).

Visuospatial and visuomotor function

Visuospatial and visuomotor function were measured with the tangle task, subdivided into 'total number correctly solved' and 'reaction time,' and did not show any significant effects, although a trend of impairment by MDMA $(F(1,12)=3.966, p=0.068)$ was observed.

\section{Attention}

Attention was assessed with the SDST task; the outcome measures were 'motor time' (see "Psychomotor function"), 'matching time' (Fig. 3) and 'total number correctly substituted.' The time required to match symbols to the corresponding numbers showed a significant MDMA and ethanol interaction $(F(1,12)=6.214, p=0.027)$. Tests for simple main effects revealed that both single-drug conditions reduced attention compared to placebo (ethanol

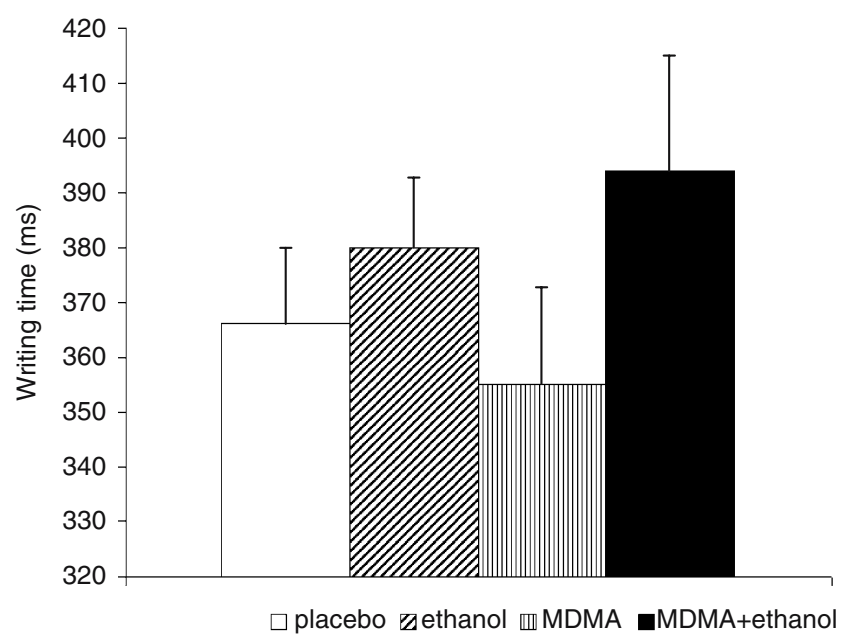

Fig. 2 Psychomotor effects: SDST writing time (mean, SEM). Ethanol increased writing times $(F(1,12)=9.295, p=0.009)$ 


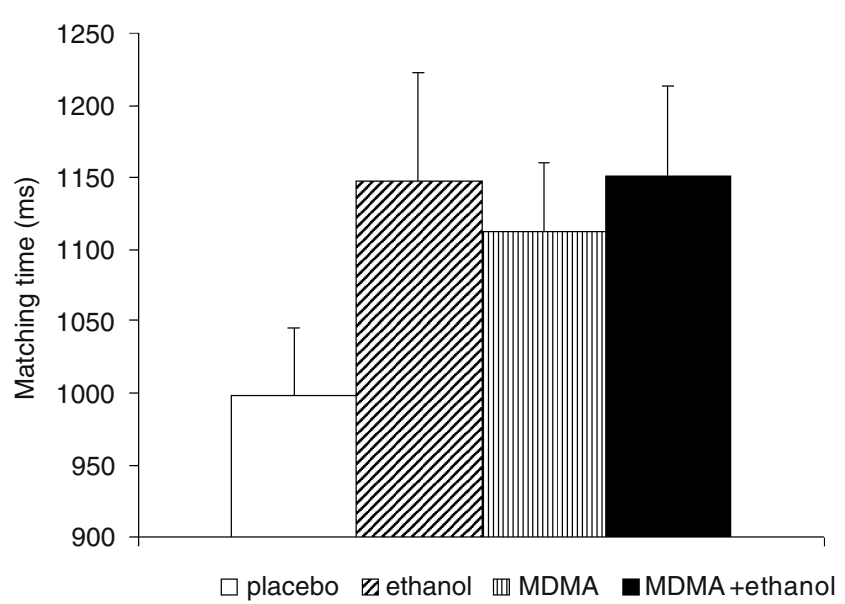

Fig. 3 Attention effects: SDST matching time, i.e. time needed for translation (mean and SEM). A significant MDMA by ethanol interaction was found $(F(1,12)=6.214, p=0.027)$

$F(1,13)=6.248, p=0.027 ;$ MDMA $F(1,13)=6.822, p=$ 0.022; see Fig. 3).

Subjective effects

Subjective effects are depicted in Fig. 4. Feelings of 'contentedness' where increased significantly by MDMA only $(F(1,12)=4.710, p=0.049)$.

A significant interaction effect $(F(1,12)=7.358, p=$ 0.018) was found for feelings of 'alertness.' Tests for simple main effects revealed that ethanol but not MDMA significantly decreased feelings of alertness compared to placebo $(F(1,13)=50.613, p<0.001)$. Feelings of 'calmness' were reduced only by MDMA $(F(1,12)=20.259$, $p=0.001$ ).

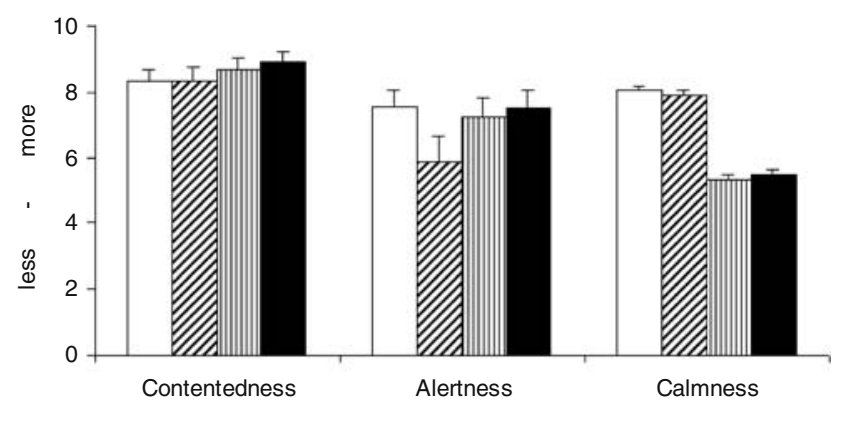

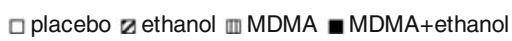

Fig. 4 Subjective effects (aggregated Bond and Lader scores, mean and SEM). Feelings of 'Contentedness' where increased significantly by MDMA only $(F(1,12)=4.710, p=0.049)$ A significant interaction effect $(F(1,12)=7.358, p=0.018)$ was found for feelings of 'Alertness.' Feelings of 'Calmness' were reduced only by MDMA $(F(1,12)=20.259, p=0.001)$

\section{Discussion}

This study demonstrates that the effects of 100-mg MDMA, commonly known as ecstasy, on cognitive function are no greater than the effects of a relatively low dose of ethanol. This is remarkable as these results suggest that the effects of 100-mg MDMA are comparable to the peak effects of two to three alcoholic beverages. Co-administration of these compounds did not result in any significant cognitive impairments beyond those observed after administration of only ethanol. The use of moderate amounts of alcohol is common in Western societies and, although impairing cognitive function, socially accepted, while ecstasy use remains very controversial. Of course, our findings only relate to the acute neuropsychological implications of ecstasy use and not to the physiological and long-term effects, which rightfully remain topics of discussion (Gouzoulis-Mayfrank and Daumann 2006a; Nutt 2006; Parrott 2007).

Drug effects observed in this placebo-controlled crossover study were moderate. Co-administration was well tolerated as indicated by the subjective scores, which were comparable to those found after single administration of MDMA. An interaction of MDMA and ethanol was found for subjective alertness scores. Ethanol, as expected, reduced subjective alertness, while MDMA co-administration reversed the reduction of subjective alertness by ethanol. In the present study, MDMA by itself did not significantly affect subjective alertness, although this effect has been consistently reported in other studies and is a well-known effect of amphetamines. However, MDMA did significantly reduce subjective calmness, i.e. subjects felt more excited after MDMA use. Probably, the Bond and Lader Mood Rating Scale is not well suited for the assessment of subjective effects of psychoactive drug effects and future studies should employ more appropriate subjective drug effect measures such as the Profile Of Mood States (de Wit et al. 2002).

When considering the results for each neuropsychological domain, executive function was not affected by any drug condition. A previous study showed impairment of executive function by ethanol but not MDMA, although ethanol impaired performance in only one out of three tests of executive function (Lamers et al. 2003). The BAC in this study was $0.3 \%$ at the time of testing compared to $0.56 \%$ in our current study, suggesting a lack of sensitivity of the test employed in the current study.

The above-mentioned previous study also reported visuospatial and visuomotor impairment by MDMA but not by ethanol. Although not significant, our current results show a similar pattern where MDMA showed a trend of impairment of visuospatial and visuomotor function, whereas ethanol did not. 
Psychomotor function was impaired only after ethanol administration (SDST motor time, see Fig. 2). The majority of studies addressed in our review of acute effects of MDMA in humans (Dumont and Verkes 2006) did not report any change in psychomotor function after MDMA either. However, increased psychomotor function after MDMA has also been found (Lamers et al. 2003; Ramaekers et al. 2006). These studies administered $75 \mathrm{mg}$ instead of $100 \mathrm{mg}$. Possibly, the effects of MDMA are biphasic, with a low dose of MDMA exhibiting more amphetamine-like effects, e.g. arousal, increasing performance, whereas higher doses may elicit more hallucinogenic effects and impair performance (Liechti et al. 2001; Solowij et al. 1992).

As mentioned above, MDMA co-administration reversed the ethanol-induced feelings of sedation, although MDMA was unable to reverse the psychomotor impairment induced by ethanol. This dissociation between subjective and objective sedation confirms previous findings by Hernandez-Lopez et al. (2002).

Several studies assessed MDMA's effect on attention using the Digit Symbol Substitution Task (DSST), although no significant effects were found (Cami et al. 2000; Farre et al. 2004; Kuypers and Ramaekers 2005). One study reported decreased DSST performance after ethanol as well as after ethanol and MDMA co-administration but no effect of MDMA (Hernandez-Lopez et al. 2002). Our findings confirm these findings to a large extent. We found no main effects of MDMA or ethanol on attention, although an interaction of ethanol and MDMA for 'matching time' (time required to match the number to the corresponding symbol) was found. Co-administration of MDMA and ethanol increased 'matching time' comparable to the increase observed after both MDMA and ethanol single administration, compliant with our hypothesis of competitive mechanisms of action of both drugs (see Fig. 3).

Studies investigating the long-term effects of MDMA consistently found memory to be affected (Verbaten 2003). In the present report, almost all memory measures showed quantitatively comparable impairment for each drug condition (see Fig. 1), although the effect of MDMA on immediate recall did not reach statistical significance. Only delayed recognition was not impaired in any drug condition. These findings suggest a deficit in the retrieval of verbal information encoded in memory, rather than impairment in the storage of information. Our findings are similar to the results of a previous study on MDMA-induced effects on memory (Kuypers and Ramaekers 2005). In this previous study, no memory impairment was observed after methylphenidate administration, a pronounced dopamine and norepinephrine releaser, suggesting the involvement of serotonin in memory impairment. Several other studies also have shown serotonin-mediated modulation of memory function through interaction with the cholinergic neurotransmitter system, although the details of this complicated interaction remain elusive (Cassel and Jeltsch 1995; GarciaAlloza et al. 2006; Meneses 2007). Generally, subjects stated that they were well aware of their impaired memory after MDMA.

BAC was on average $0.56 \%$. At this level, driving is prohibited by law in many European countries because of its interference with normal functioning. Although the effects were moderate, ethanol impaired cognitive performance in various tests. Similar moderate effects were observed with MDMA $100 \mathrm{mg}$, considered to be slightly above the average recreational dose (Tanner-Smith 2006). This might be considered surprising for a drug with reported robust subjective stimulating and hallucinogenic properties. However, because the effects caused by a single dose of 100-mg MDMA were comparable to the effects of a BAC of $0.56 \%$, this dose should by inference be considered unacceptable in motorised traffic.

Arguably, the moderate drug effects as found in this study could be explained by 'missing' the time of the maximal drug effects. Although the average MDMA blood concentration reported here $(196 \mu \mathrm{g} / \mathrm{L})$ is comparable to Cmax of 100-mg MDMA $(199.8 \mu \mathrm{g} / \mathrm{L})$ as reported by de la Torre et al. (2000), MDMA concentration was assessed at the end of the testing procedure. However, HernandezLopez et al. (2002) found significant effects at $60 \mathrm{~min}$ as well as $90 \mathrm{~min}$ after drug administration, arguing against the suggestion of 'missing' peak drug effects.

The circumstances in which these substances are normally used cannot be fully recreated in the laboratory and this may have suppressed the effects of both substances. It is not unlikely that these substances show enhanced effects when tested under typical circumstances and surroundings. Recently, Parrott et al. (2006) concluded that the increase in physical activity and body temperature typically experienced when using MDMA enhance MDMA effects. Ball et al. (2006) demonstrated that a familiar surrounding increased MDMA-induced locomotor response as well as single-neuron activity in rats, compared to unfamiliar surroundings. Therefore, the psychosocial context in which MDMA is used, along with the different expectations and behaviour, probably influences its effects (Sumnall et al. 2006). It is unlikely, however, that this affects the quality of the interactions of MDMA and ethanol.

In conclusion, co-administration of MDMA and ethanol did not impair cognitive function significantly more than MDMA or ethanol administration alone. The most prominent effect of (co-)administration of MDMA and ethanol was an impairment of memory. Ethanol also impaired psychomotor function. Although the impairment of performance by each drug condition was relatively moderate, this 
significant impairment of cognitive function should be considered intolerable in motorised traffic and other cognitively demanding situations as confirmed by previous research and as defined by law. However, the effects of these drugs in the concentrations used in the present study on established neuropsychological tests appear to be smaller than one would assume based on their reputation.

Acknowledgements This research was supported by a grant of ZonMW (31000062), the Netherlands and complies with current laws. We thank Jan Leytens for his assistance during the experiments, John van den Bercken for statistical advice and Hans van Bree for proofreading the manuscript.

Open Access This article is distributed under the terms of the Creative Commons Attribution Noncommercial Licence which permits any noncommercial use, distribution, and reproduction in any medium, provided the original author(s) and source are credited.

\section{References}

Amatsaleh AA, Dumont GJ, Schoemaker RC, van Gerven JM (2006a) Optimisation of a novel method for alcohol infusion by clamping of breath alcohol concentration. Br J Clin Pharmacol $61: 475$

Amatsaleh AA, Schoemaker RC, van Gerven JM (2006b) Investigation of the effects of ethanol on central nervous system parameters in healthy volunteers. Br J Clin Pharmacol 61:626

Ammons RB (1951) Effect of distribution of practice on rotary pursuit hits. J Exp Psychol 41:17-22

Baker EL, Letz R (1986) Neurobehavioral testing in monitoring hazardous workplace exposures. J Occup Med 28:987-990

Ball KT, Budreau D, Rebec GV (2006) Context-dependent behavioural and neuronal sensitization in striatum to MDMA (ecstasy) administration in rats. Eur J Neurosci 24:217-228

Barrett SP, Gross SR, Garand I, Pihl RO (2005) Patterns of simultaneous polysubstance use in Canadian rave attendees. Subst Use Misuse 40:1525-1537

Bittner AC Jr, Carter RC, Kennedy RS, Harbeson MM, Krause M (1986) Performance Evaluation Tests for Environmental Research (PETER): evaluation of 114 measures. Percept Mot Skills 63:683-708

Bond AJ, James DC, Lader MH (1974) Physiological and psychological measures in anxious patients. Psychol Med 4:364-373

Cami J, Farre M, Mas M, Roset PN, Poudevida S, Mas A, San L, de la Torre R (2000) Human pharmacology of 3,4-methylenedioxymethamphetamine ("ecstasy"): psychomotor performance and subjective effects. J Clin Psychopharmacol 20:455-466

Cassel JC, Jeltsch H (1995) Serotonergic modulation of cholinergic function in the central nervous system: cognitive implications. Neuroscience 69:1-41

Colado MI, O'Shea E, Green AR (2004) Acute and long-term effects of MDMA on cerebral dopamine biochemistry and function. Psychopharmacology 173:249-263

de la Torre R, Farre M, Ortuno J, Mas M, Brenneisen R, Roset PN, Segura J, Cami J (2000) Non-linear pharmacokinetics of MDMA ('ecstasy') in humans. Br J Clin Pharmacol 49:104-109

de la Torre R, Farre M, Roset PN, Pizarro N, Abanades S, Segura M, Segura J, Cami J (2004) Human pharmacology of MDMApharmacokinetics, metabolism, and disposition. Ther Drug Mon 26:137-144 de Wit H, Enggasser JL, Richards JB (2002) Acute administration of d-amphetamine decreases impulsivity in healthy volunteers. Neuropsychopharmacology 27:813-825

Dumont GJ, Verkes RJ (2006) A review of acute effects of 3,4methylenedioxymethamphetamine in healthy volunteers. J Psychopharmacol 20:176-187

Farre M, de la Torre R, Mathuna BO, Roset PN, Peiro AM, Torrens M, Ortuno J, Pujadas M, Cami J (2004) Repeated doses administration of MDMA in humans: pharmacological effects and pharmacokinetics. Psychopharmacology 173:364-375

Garcia-Alloza M, Zaldua N, ez-Ariza M, Marcos B, Lasheras B, Javier Gil-Bea F, Ramirez MJ (2006) Effect of selective cholinergic denervation on the serotonergic system: implications for learning and memory. J Neuropathol Exp Neurol 65:10741081

Gouzoulis-Mayfrank E, Daumann J (2006a) Neurotoxicity of methylenedioxyamphetamines (MDMA; ecstasy) in humans: how strong is the evidence for persistent brain damage. Addiction 101:348-361

Gouzoulis-Mayfrank E, Daumann J (2006b) The confounding problem of polydrug use in recreational ecstasy/MDMA users: a brief overview. J Psychopharmacol 20:188-193

Green AR, Mechan AO, Elliott JM, O'Shea E, Colado MI (2003) The pharmacology and clinical pharmacology of 3,4-methylenedioxymethamphetamine (MDMA, "ecstasy"). Pharmacol Rev $55: 463-508$

Gross SR (2002) Ecstasy and drug consumption patterns: a Canadian rave population study. Can J Psychiatry 47:546-551

Hege SG, Ellinwood EH Jr., Wilson WH, Helligers CA, Graham SM (1997) Psychomotor effects of the anxiolytic abecarnil: a comparison with lorazepam. Psychopharmacology 131:101-107

Hernandez-Lopez C, Farre M, Roset PN, Menoyo E, Pizarro N, Ortuno J, Torrens M, Cami J, de la Torre R (2002) 3,4methylenedioxymethamphetamine (ecstasy) and alcohol interactions in humans: psychomotor performance, subjective effects, and pharmacokinetics. J Pharmacol Exp Ther 300:236-244

Kaplan E, Fein D, Morris R, Delis D (1991) Wais-R as a neuropsychological instrument. Psychological Corporation, San Antonio

Kirk RE (1995) Experimental design: procedures for the behavioral sciences, 3rd edn. Brooks/Cole, Pacific Grove

Kuypers KP, Ramaekers JG (2005) Transient memory impairment after acute dose of $75 \mathrm{mg}$ 3.4-Methylene-dioxymethamphetamine. J Psychopharmacol 19:633-639

Kuypers KP, Ramaekers JG (2007) Acute dose of MDMA (75 mg) impairs spatial memory for location but leaves contextual processing of visuospatial information unaffected. Psychopharmacology 189:557-563

Kuypers KP, Samyn N, Ramaekers JG (2006) MDMA and alcohol effects, combined and alone, on objective and subjective measures of actual driving performance and psychomotor function. Psychopharmacology 187:467-475

Kuypers KP, Wingen M, Samyn N, Limbert N, Ramaekers JG (2007) Acute effects of nocturnal doses of MDMA on measures of impulsivity and psychomotor performance throughout the night. Psychopharmacology 192:111-119

Lamers CTJ, Ramaekers JG (2001) Visual search and urban city driving under the influence of marijuana and alcohol. Hum Psychopharmacol Clin Exp 16:393-401

Lamers CTJ, Ramaekers JG, Muntjewerff ND, Sikkema KL, Samyn N, Read NL, Brookhuis KA, Riedel WJ (2003) Dissociable effects of a single dose of ecstasy (MDMA) on psychomotor skills and attentional performance. J Psychopharmacol 17:379-387

Liechti ME, Gamma A, Vollenweider FX (2001) Gender differences in the subjective effects of MDMA. Psychopharmacology 154:161-168 
Liechti ME, Vollenweider FX (2000) The serotonin uptake inhibitor citalopram reduces acute cardiovascular and vegetative effects of 3,4-methylenedioxymethamphetamine ('ecstasy') in healthy volunteers. J Psychopharmacol 14:269-274

Liechti ME, Vollenweider FX (2001) Which neuroreceptors mediate the subjective effects of MDMA in humans? A summary of mechanistic studies. Hum Psychopharmacol Clin Exp 16:589-598

Maxwell SE, Delaney HD (2004) Designing experiments and analyzing data: a model comparison perspective, 2nd edn. Lawrence Erlbaum, Mahwah

Meneses A (2007) Do serotonin(1-7) receptors modulate short and long-term memory. Neurobiol Learn Mem 87:561-572

Mlinar B, Corradetti R (2003) Endogenous 5-HT, released by MDMA through serotonin transporter- and secretory vesicle-dependent mechanisms, reduces hippocampal excitatory synaptic transmission by preferential activation of 5-HT1B receptors located on CA1 pyramidal neurons. Eur J Neuroscience 18:1559-1571

Nichols DE, Oberlender R (1990) Structure-activity relationships of MDMA and related compounds: a new class of psychoactive drugs. Ann N Y Acad Sci 600:613-623

Nutt DJ (2006) A tale of two Es. J Psychopharmacol 20:315-317

Parrott AC (2001) Human psychopharmacology of Ecstasy (MDMA): a review of 15 years of empirical research. Hum Psychopharmacol 16:557-577

Parrott AC (2007) Ecstasy versus alcohol: Tolstoy and the variations of unhappiness. J Psychopharmacol 21:3-6

Parrott AC, Rodgers J, Buchanan T, Ling J, Heffernan T, Scholey AB (2006) Dancing hot on ecstasy: physical activity and thermal comfort ratings are associated with the memory and other psychobiological problems reported by recreational MDMA users. Hum Psychopharmacol 21:285-298

Pifl C, Drobny H, Reither H, Hornykiewicz O, Singer EA (1995) Mechanism of the dopamine-releasing actions of amphetamine and cocaine-plasmalemmal dopamine transporter versus vesicular monoamine transporter. Mol Pharmacolog 47:368-373

Ramaekers JG, Kuypers KP, Samyn N (2006) Stimulant effects of 3,4methylenedioxymethamphetamine (MDMA) $75 \mathrm{mg}$ and methylphenidate $20 \mathrm{mg}$ on actual driving during intoxication and withdrawal. Addiction 101:1614-1621

Riley SC, James C, Gregory D, Dingle H, Cadger M (2001) Patterns of recreational drug use at dance events in Edinburgh, Scotland. Addiction 96:1035-1047

Sabbe B, Hulstijn W, van HJ, Tuynman-Qua HG, Zitman F (1999) Retardation in depression: assessment by means of simple motor tasks. J Affect Disord 55:39-44
Solowij N, Hall W, Lee N (1992) Recreational MDMA use in Sydney: a profile of 'ecstasy' users and their experiences with the drug. $\mathrm{Br}$ J Addict 87:1161-1172

Sumnall HR, Cole JC, Jerome L (2006) The varieties of ecstatic experience: an exploration of the subjective experiences of ecstasy. J Psychopharmacol 20:670-682

Suzdak PD, Schwartz RD, Skolnick P, Paul SM (1988) Alcohols stimulate gamma-aminobutyric acid receptor-mediated chloride uptake in brain vesicles: correlation with intoxication potency. Brain Res 444:340-345

Tancer M, Johanson CE (2007) The effects of fluoxetine on the subjective and physiological effects of 3,4-methylenedioxymethamphetamine (MDMA) in humans. Psychopharmacology 189:565-573

Tanner-Smith EE (2006) Pharmacological content of tablets sold as "ecstasy": results from an online testing service. Drug Alcohol Depend 83:247-254

Vakil E, Blachstein H (1993) Rey Auditory-Verbal Learning Test: structure analysis. J Clin Psychol 49:883-890

Verbaten MN (2003) Specific memory deficits in ecstasy users? The results of a meta-analysis. Hum Psychopharmacol Clin Exp $18: 281-290$

Verkes RJ, Gijsman HJ, Pieters MSM, Schoemaker RC, de Visser S, Kuijpers M, Pennings EJM, de Bruin D, Van de Wijngaart G, van Gerven JMA, Cohen AF (2001) Cognitive performance and serotonergic function in users of ecstasy. Psychopharmacology 153:196-202

Vollenweider FX, Liechti ME, Gamma A, Greer G, Geyer M (2002) Acute psychological and neurophysiological effects of MDMA in humans. J Psychoactive Drugs 34:171-184

Wechsler D (1981) The psychometric tradition-developing the Wechsler Adult Intelligence Scale. Contemp Educ Psychol 6:82-85

Wezenberg E, Hulstijn W, Sabbe B, Ruigt GS, Verkes RJ (2004) Psychomotor and cognitive effects of lorazepam and D-amphetamine in healthy subjects. Development of sensitive screening tests of psychomotor- and cognitive functions.. Eur Neuropsychopharmacol 14:S362

Wezenberg E, Verkes RJ, Sabbe BG, Ruigt GS, Hulstijn W (2005) Modulation of memory and visuospatial processes by biperiden and rivastigmine in elderly healthy subjects. Psychopharmacology 181:582-594

Winstock AR, Griffiths P, Stewart D (2001) Drugs and the dance music scene: a survey of current drug use patterns among a sample of dance music enthusiasts in the UK. Drug Alcohol Depend 64:9-17 